\title{
MIXED TOTAL PULMONARY VENOUS DRAINAGE: STILL A SURGICAL CHALLENGE
}

Ralph E. Delius, MD

Marc R. de Leval, MD

Martin J. Elliott, MD

Jaroslav Stark, MD
Ojective: The aim of this report is to review the surgical experience of a single institution with a relatively large series of patients with mixed total pulmonary venous drainage. Patient population: Between January 1, 1971, and December 31, 1994, 232 patients with total pulmonary venous drainage underwent surgical correction. Twenty of these patients $(8.6 \%)$ had mixed type total pulmonary venous drainage. Ages at operation ranged from 1 day to $\mathbf{4 6}$ months, with a median of 2.3 months. Results: Both cardiac catheterization and echocardiography were performed before operation in 12 patients. Four patients underwent only cardiac catheterization, and another four patients underwent only echocardiography. The sensitivity and specificity for catheterization were $94 \%$ and $99 \%$, respectively; they were $31 \%$ and $100 \%$, respectively, for echocardiography. Severe pulmonary venous obstruction was present in three patients, all of whom underwent emergency operation. Three patients $(15 \%)$, all of whom had preoperative pulmonary venous obstruction, died after operation. There were two late deaths, one of pulmonary vein stenosis and the other of probable pulmonary hypertension. The actuarial survival at 10 years was $73 \%$ for all patients; patients who survived the initial operation had a 10-year survival of $87 \%$. Conclusion: The diagnosis of mixed total pulmonary venous drainage can be difficult to establish by echocardiography or at the time of operation. For patients in stable condition, cardiac catheterization may be considered if fewer than three pulmonary veins are identified by echocardiography. Pulmonary venous obstruction is relatively infrequent in this group of patients but when present impacts patient survival significantly. The long-term results with this lesion are excellent. (J Thorac Cardiovasc Surg 1996;112:1581-8)
$T$ otal anomalous pulmonary venous drainage (TAPVD) is a rare congenital heart lesion that has long challenged the surgeon dealing with congenital heart defects. Several recent reports however, have documented improving results with time for this lesion. ${ }^{1-4}$ Early and accurate diagnosis, prompt repair, and improved control of postopera-

From the Great Ormond Street Hospital NHS Trust, London, United Kingdom.

Read at the Seventy-sixth Annual Meeting of The American Association for Thoracic Surgery, San Diego, Calif., April 28-May 1, 1996.

Received for publication May 3, 1996; revisions requested June 21, 1996; revisions received July 24, 1996; accepted for publication July 29, 1996.

Reprint requests: Marc R. de Leval, MD, FRCS, Cardiothoracic Unit, Great Ormond Street Hospital for Children, London WC1N 3JH, United Kingdom.

Copyright (C) 1996 by Mosby-Year Book, Inc.

0022-5223/96 \$5.00+0 $\mathbf{1 2 / 6 / 7 6 9 1 7}$ tive pulmonary hypertension have been emphasized as critical factors in the improving results seen with this lesion. Some reports show an increased surgical risk for the infradiaphragmatic anatomic subtype of TAPVD, ${ }^{5,6}$ although this may no longer be a risk factor. $^{3,7-9}$ In several series, the mortality risk for the mixed anatomic subtype also appears to be increased, but this variant is relatively infrequent and most reports contain few patients with mixed TAPVD. ${ }^{10,11}$ Consequently, it has been difficult to derive any conclusions regarding surgical mortality rates, risk of reoperation, late survival and functional status, or principles of repair for this lesion. Treatment of patients with mixed TAPVD is particularly challenging with respect to preoperative diagnosis and the technical aspects of surgical repair, especially for patients with mixed TAPVD who do not have a horizontal venous confluence. This review retrospectively analyzes a relatively large series of patients with this uncommon variant of TAPVD. 

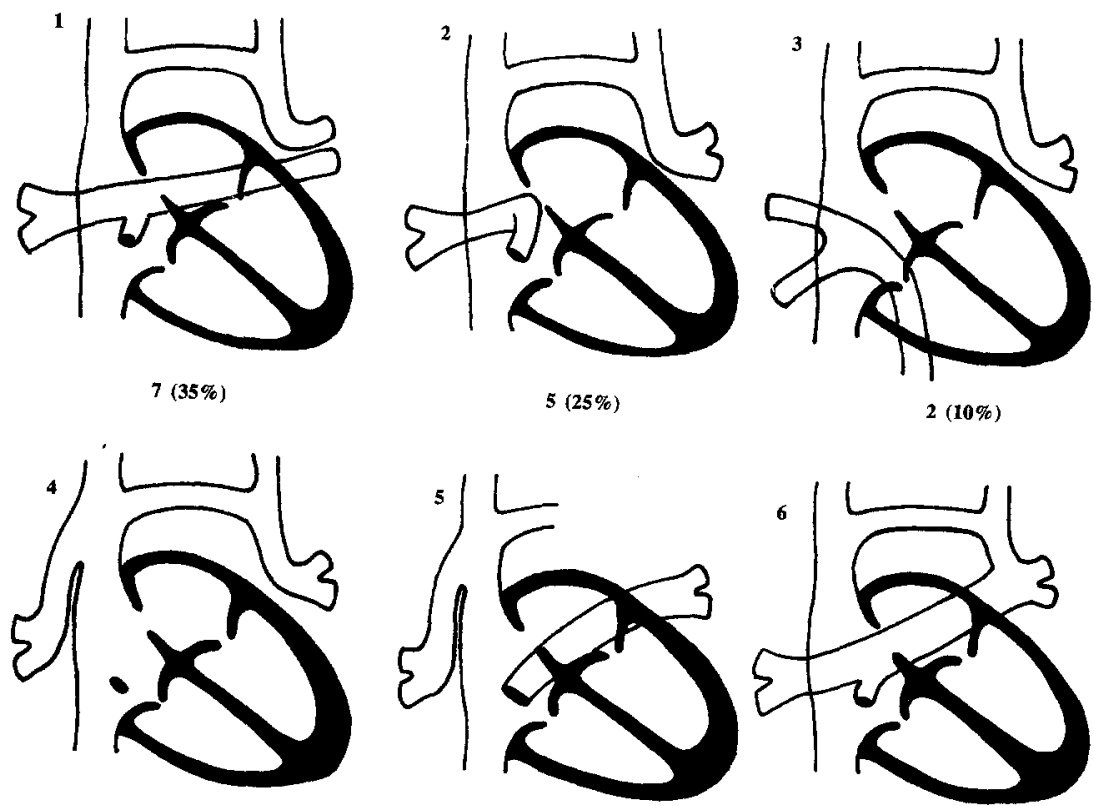

$1(5 \%)$
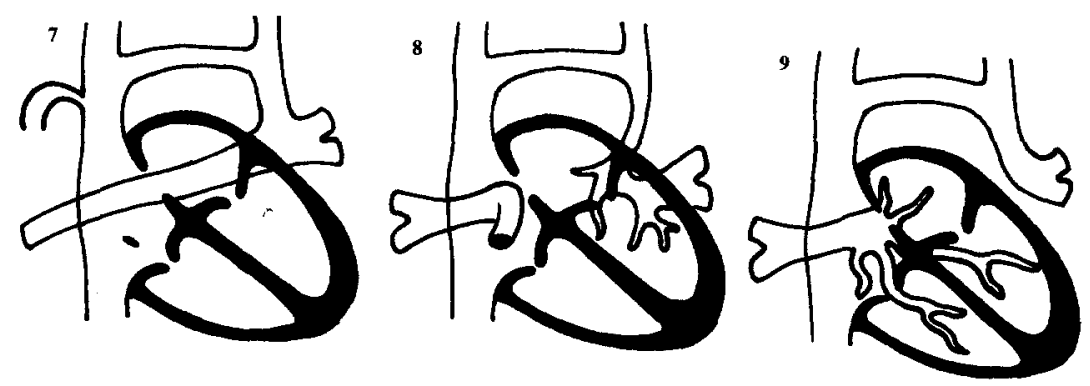

$1(5 \%)$

$1(5 \%)$

$1(5 \%)$

Fig. 1. Schematic representation of the morphologic variants of mixed TAPVD. Number of patients and frequency are listed below each illustration. See text for detailed descriptions of variants 1 through 9 .

\section{Methods}

Patient population. Between January 1971 and December 1994, 232 patients with the diagnosis of TAPVD underwent surgical repair at the Great Ormond Street Hospital for Children. The mixed anatomic type of TAPVD was present in 20 patients $(8.6 \%)$. These 20 patients, 12 male and eight female, constitute the study group. Weight at surgical repair ranged from 2.3 to $16 \mathrm{~kg}$ (median $4.2 \mathrm{~kg}$ ). Age at operation ranged from 1 day to 46 months, with a median of 2.3 months.

Preoperative diagnostic studies. Preoperative diagnostic studies included both cardiac catheterization and echocardiography in 12 patients. Echocardiography was the only diagnostic study in four cases, and cardiac catheterization was the sole diagnostic study in another four cases. All patients seen since 1981 have undergone preoperative echocardiography. Balloon atrial septostomy was per- formed at cardiac catheterization in two patients. For patients who underwent preoperative cardiac catheterization, systolic pulmonary artery pressures ranged from 20 to $80 \mathrm{~mm} \mathrm{Hg}$ (mean $53 \mathrm{~mm} \mathrm{Hg}$ ). The ratio of systolic pulmonary artery pressure to systemic systolic pressure ranged from 0.22 to 1.2 (mean 0.64 ). Preoperative systemic oxygen saturation ranged from $65 \%$ to $95 \%$ (mean $77 \%$ ).

Morphology. The anatomy and frequency of the mixed morphologic subtypes can be seen in Fig. 1. Most patients had a combination of the more common anatomic subtypes of TAPVD, such as the right pulmonary veins draining into the coronary sinus and one (Fig. 1, 1) or both (Fig. 1,2) left pulmonary veins draining into a vertical vein. Two patients had pulmonary veins on the right side connecting to a descending vein that ultimately drained into a vein below the diaphragm. Both veins on the left 
side connected to a vertical vein, which in turn drained into the innominate vein (Fig. 1, 3). Two patients had right-sided pulmonary veins draining into the superior vena cava, with left-sided veins draining into a vertical vein (Fig. 1,4) or the coronary sinus (Fig. 1, 5). One patient had a typical coronary sinus type defect that also connected to a vertical vein (Fig. 1, 6); this vein was actually a left superior vena cava because it connected the coronary sinus and left innominate vein. Another patient had an obstructed right upper vein draining into the superior vena cava, with the remaining veins connected to a horizontal vein that ultimately drained into a vertical vein (Fig. 1, 7). Two patients had an unusual plexus of veins behind the pericardium, one arising from the left lung (Fig. 1,8) and the other arising from the right lung (Fig. 1, 9).

Associated cardiac lesions. A perimembranous ventricular septal defect was present in three patients. Pulmonary valvular stenosis was noted in one patient.

Operations. The earliest patient in the series had the repair performed through a bilateral thoracotomy. All the remaining patients had median sternotomies. Early in the series, hypothermia was accomplished by a combination of surface cooling and cardiopulmonary bypass; since 1984 , however, bypass alone has been used for cooling. Patients were cooled to $18^{\circ}$ to $22^{\circ} \mathrm{C}$. Cardioplegia was used in the 14 patients who underwent surgical repair after 1980 . Intermittent ischemic arrest was used before 1980. The mean cardiopulmonary bypass time was 85 minutes (range 62 to 106 minutes). Circulatory arrest was used in 16 patients. The mean length of circulatory arrest was 40 minutes, with a range of 29 to 60 minutes.

In terms of surgical management, the patients could be conceptually divided into two groups: (1) " $3+1$," in which three veins drained into the coronary sinus or horizontal venous confluence, with the remaining vein draining at another site (Fig. 1, 1, 6, and 7), and (2) "2 + 2 ," where the veins of each side connected with systemic venous drainage at separate sites (Fig. 1,2, 3, 4, 5, 8, and 9).

For the $3+1$ group of patients, the drainage of the three pulmonary veins into the coronary sinus or horizontal venous confluence was surgically managed by standard surgical techniques. Patients with a coronary sinus-type defect had a portion of the atrial septum between the coronary sinus and atrial septal defect resected, with wide opening of the foramen ovale or secundum atrial septal defect and closure of the resulting defect with a pericardial or Dacron polyester fabric patch. ${ }^{12}$ If a horizontal venous confluence was present, an anastomosis with the posterior aspect of the left atrium was created with a running polypropylene suture. A variety of approaches were used for the remaining isolated pulmonary vein. In three patients with drainage of the left upper pulmonary vein into the left innominate vein (Fig. 1, l), this isolated lobar vein was left uncorrected. The remaining patients with this drainage connection had the left upper vein anastomosed to the left atrial appendage. In one case (Fig. 1, 7), an isolated right upper pulmonary vein draining into the superior vena cava was obstructed. The diagnosis of obstruction of this vein was established by chest radiography, which demonstrated pulmonary edema restricted to the right upper lobe. The site of drainage into the superior vena cava was high, and the vein could not be connected with the left atrium. This obstructed vein was reimplanted into the superior vena cava.

Patients with the $2+2$ type of drainage posed particular problems because they lacked a horizontal venous confluence behind the heart; establishing connection between the pulmonary veins and the left atrium required a variety of techniques. Vertical veins on the left side were anastomosed to the left atrial appendage. A vascular clamp was placed across the base of the left atrial appendage. The appendage was then amputated obliquely, leaving a wide opening for the anastomosis. Any remaining trabeculae were then resected. The vertical vein was ligated and divided as close to the innominate vein as possible and then mobilized to the hilum. A generous opening was made in the pericardium to create a wide passage for the vein and to prevent kinking. The end of the vertical vein was then beveled and anastomosed to the left atrial appendage with a running polypropylene suture.

Right-sided pulmonary veins were managed by conventional techniques. Veins draining into the superior vena cava (Fig. 1, 4 and 5) were connected to the left atrium by a baffle. Patients with a descending vein (Fig. 1,4) had the vein widely mobilized and anastomosed to the back of the left atrium with a running polypropylene suture. The distal descending vein was ligated in both cases; however, in one patient the right lower lobe vein was inadvertently ligated, with resulting venous infarction of the right lower lobe. Right lower lobectomy was performed on the second postoperative day. This patient's subsequent course was uncomplicated. Patients with the right veins draining into the coronary sinus (Fig. 1,2 and 8) were managed by the technique described previously.

In one patient, the right pulmonary veins drained into the coronary sinus and the left pulmonary veins drained into a plexus of veins behind the pericardium (Fig. 1,8). The pericardium was opened and the largest vein in the plexus was anastomosed to the posterior aspect of the left atrium. In another patient, the right pulmonary veins drained into a retrocardiac plexus (Fig. 1,9). The largest vein in the plexus was opened, but extensive thrombus was found. A thrombectomy was attempted unsuccessfully. No patent veins of a satisfactory size could be identified in the plexus. Postoperative angiocardiography demonstrated sluggish venous drainage from the right lung. The exact site of pulmonary venous drainage into the systemic venous system of this patient could not be identified.

Statistics. Survival was estimated by actuarial analysis. Sensitivity and specificity of echocardiography and catheterization were determined by the standard equations. ${ }^{13}$ Intraoperative findings were used as the gold standard for these determinations.

\section{Results}

Survival. The operative mortality was $15 \%$ ( $3 /$ 20). All patients who died were acutely ill before operation and underwent emergency operation in response to pulmonary venous obstruction. None of the other patients in this series had clinically apparent 
pulmonary venous obstruction; each of these patients had cyanosis. One patient died of multiple organ failure after a protracted course in the intensive care unit. At autopsy, this patient was found to have severe right ventricular hypertrophy and histologic evidence of pulmonary hypertension. The other two patients died on the day of operation, one of a pulmonary hypertensive crisis and another of low cardiac output; pulmonary hypertension may have been a contributing cause of death in this latter patient.

There were two late deaths, one during cardiac catheterization 4 months after operation to evaluate pulmonary vein stenosis caused by intimal hyperplasia. This patient (Fig. 1,6) had a coronary sinus type repair and ligation of a left vertical vein. Another patient died at home 6 weeks after operation, presumably of pulmonary hypertension although this was not clearly established. Actuarial survival at 10 years was $73 \%$ ( $70 \%$ confidence limit $63 \%$ to $85 \%$ ) for all patients (Fig. 2, $A$ ). For operative survivors, 10 -year actuarial survival was $87 \%(70 \%$ confidence limit $74 \%$ to $99 \%$; Fig. $2, B$ ).

Diagnosis. The correct diagnosis and an accurate description of anatomy were obtained before operation for 15 of 16 patients $(97 \%)$ who underwent preoperative catheterization and angiocardiography. In one patient, a left upper pulmonary vein draining into a vertical vein was undetected. There was also one patient whose diagnosis was mixedtype TAPVD by catheterization, but both operative findings and postoperative catheterization failed to confirm this diagnosis. The sensitivity of catheterization was $94 \%$ and its specificity was $99 \%$.

Of 16 patients who underwent preoperative echocardiography, the correct diagnosis and description of anatomy were determined in only five cases $(31 \%)$. In one case, the diagnosis of TAPVD was missed altogether. In the other cases, the diagnosis of TAPVD was established but the presence of the mixed type was not realized. There were no known false-positive echocardiographic diagnoses of mixed-type TAPVD. The sensitivity and specificity of echocardiography for mixed TAPVD were $31 \%$ and $100 \%$, respectively.

Postoperative course. All patients required postoperative inotropic support. The mean duration of ventilator support was 7 days (range 2 to 27 days), and the average postoperative stay in the intensive care unit was 7 days (range 3 to 32 days).

Two patients early in our experience had postoperative seizures. One patient was cooled to a nasopharyngeal temperature of $10^{\circ} \mathrm{C}$ and the other was cooled to $15^{\circ} \mathrm{C}$, both of which were lower temperatures than used in any other patient in this series; it is unknown whether this may have contributed to the seizures in these patients. The circulatory arrest times for these two patients were 43 and 55 minutes, respectively. Two patients had acute postoperative renal failure that necessitated temporary peritoneal dialysis. One patient had a case of Escherichia coli infection that responded to antibiotics.

Reoperations. One patient mentioned earlier underwent a right lower lobectomy on the second postoperative day as a result of inadvertent ligation of the right lower lobe vein. There were no late reoperations.

Late functional status. Complete follow-up was available for all but one patient; this patient was no longer available for follow-up 16 years after operation. All patients were followed up by local cardiologists and periodically underwent echocardiography; the intervals between echocardiographic follow-up varied according to local custom. The mean length of follow-up was 9 years, with a range of 2 months to 21 years. All survivors are in New York Heart Association functional class I and are free of medications. The three patients who had drainage of the left upper pulmonary vein left uncorrected did not have any clinically apparent sequelae. One of these patients had a postoperative cardiac catheterization, by which the ratio of systemic to pulmonary resistance was documented to be less than 1.5:1.

\section{Discussion}

TAPVD is a rare congenital heart defect, accounting for only $1 \%$ to $2 \%$ of all cardiac defects. ${ }^{14}$ Appreciation of the embryology of pulmonary venous development is necessary to understand the morphologic variants of TAPVD. Early in development, the lung buds are enmeshed in a splanchnic plexus that communicates superiorly with the anterior cardinal veins and inferiorly with the umbilicalvitelline system. There is no connection with the heart at this stage. Communication between the heart and the pulmonary vascular bed depends on evagination of a common pulmonary vein from the sinoatrial portion of the heart. When connection is made between the pulmonary vasculature and the heart, the communications with the cardinal and umbilical-vitelline systems are lost. ${ }^{13}$ One classification system of TAPVD emphasizes the embryologic origins of the anomalous venous connections: (1) drainage to the right atrium, (2) drainage to the 

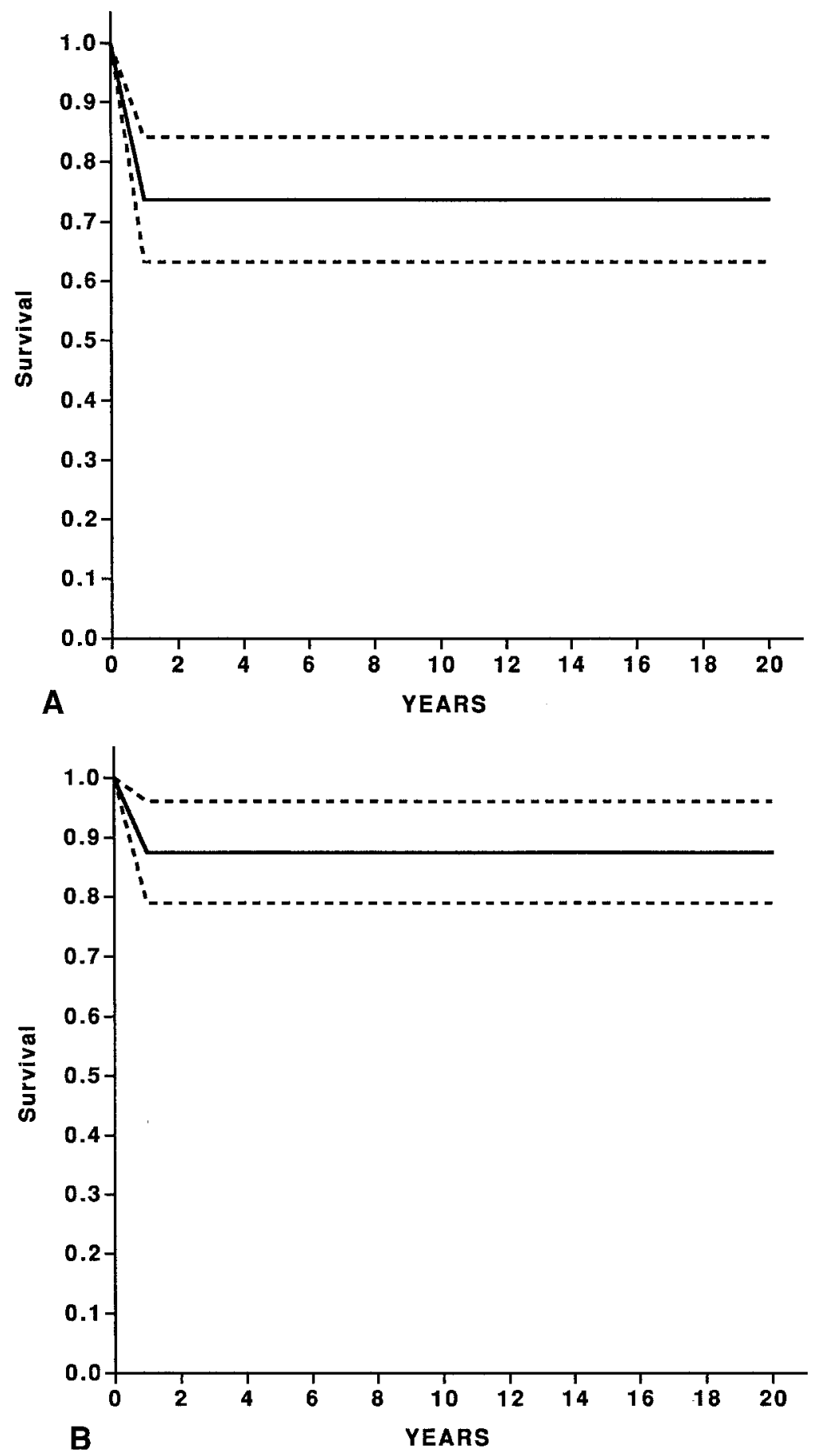

Fig. 2. A, Actuarial estimate of survival for all patients undergoing surgical repair of mixed TAPVD. Dotted lines represent $70 \%$ confidence limits. B, Actuarial estimate of survival for patients surviving operation for mixed TAPVD. Dotted lines represent $70 \%$ confidence limits.

right common cardinal system (superior vena cava or azygous vein), (3) drainage to the left common cardinal system (left innominate vein or coronary sinus), and (4) drainage to the umbilical-vitelline system (portal vein, ductus venosus, and so on)..$^{15}$ Although this classification clarifies the embryology of TAPVD, the most clinically useful classification system, that of Darling, Rothney, and Craig, ${ }^{16}$ cat- 
egorizes the various forms of this lesion by site of connection of the anomalous pulmonary venous pathways. The supracardiac type (type I), which is the most common, has an anomalous connection at the supracardiac level, usually the innominate vein but occasionally at the superior vena cava or azygous vein. Type II lesions include all connections at the cardiac level, usually into the coronary sinus. Patients can also have anomalous infracardiac connections (type III). The mixed type (type IV) involves anomalous connections at two or more separate sites. The mixed type is the least common type, constituting $5 \%$ to $10 \%$ of patients with TAPVD in large series. ${ }^{3,17,18}$ Principles of management and surgical repair of mixed TAPVD have been difficult to define because of the small number of patients in most surgical series.

Two patients in this series had an extremely unusual form of mixed TAPVD, with a retrocardiac plexus behind the pericardium draining the right lung in one case and the left lung in the other. In both cases, echocardiography failed to identify any pulmonary veins on the respective sides. The embryology of this defect is uncertain, but the plexus may represent persistence of the splanchnic plexus that enmeshed the lungs buds early in development.

The horizontal venous confluence present in most cases of extracardiac TAPVD has been called "the surgeon's friend" 18 because in most cases this confluence can be anastomosed to the back of the left atrium relatively easily. In mixed TAPVD, however, the horizontal venous confluence is frequently $a b-$ sent altogether. ${ }^{18}$ The surgical approach to patients with mixed TAPVD can be simplified by classifying patients as having a $3+1$ connection or a $2+2$ connection. It is important for the surgeon to confirm the drainage pattern of all four pulmonary veins during operation. Patients with a $3+1$ connection can have the coronary sinus or horizontal venous confluence connected to the left atrium by the well-described techniques used for the more common forms of TAPVD. ${ }^{1-9}$ Management of the remaining pulmonary vein must be individually tailored. If there is evidence that the isolated vein is obstructed, creation of a connection to the left atrium is mandatory. If the vein is not obstructed, a variety of options are available. The vein may be left uncorrected. This will leave a small left-to-right shunt, because when a single pulmonary vein is anomalously connected, the anomalously draining blood flow is about $20 \%$ of total pulmonary blood flow. ${ }^{19}$ Patients who have a single anomalously connected vein usually do not come to medical attention and are considered to have an excellent prognosis. ${ }^{14}$ There have, however, been isolated reports of pulmonary vascular obstructive disease developing as a consequence of the left-to-right shunt present in patients with a single anomalous pulmonary vein. ${ }^{20,21}$ Some groups have therefore advocated repair of all anomalously draining single pulmonary veins. ${ }^{20,22,23}$

A novel approach suggested by Brantigan ${ }^{24}$ for patients with isolated anomalous lobar venous drainage is removal of the lobe that is anomalously connected. Lobectomy is well tolerated in infants, as demonstrated by the patient in this series who required lobectomy for venous infarction. There is a potential for pulmonary growth after lobectomy because alveolar development continues until approximately 7 years of age, and a theoretic argument could be made that lobectomy may be preferable to leaving a left-to-right shunt, which in some circumstances could be greater than 1.5 to 1 .

Patients with a $2+2$ pattern of drainage can be conceptually approached as having bilateral partial anomalous pulmonary venous drainage. Techniques for establishment of connection between right pulmonary veins and the left atrium have been well described. ${ }^{25}$ Isolated anomalous venous drainage on the left side is much less common than on the right. Most techniques of connecting left-sided veins involve an anastomosis to the left atrial appendage, as first described by Kirklin. ${ }^{26}$ Concerns have arisen regarding the patency of an anastomsis to the left atrial appendage because this structure is heavily trabeculated. ${ }^{27}$ The long-term patency of this anastomosis has not been established. The excellent long-term outcome in patients with this anastomotic connection in this series as well as others, ${ }^{22}$ however, suggests that clinically apparent obstruction is uncommon.

The correct preoperative diagnosis and an accurate description of anatomy are important for surgical management of patients with mixed TAPVD. In most cases of TAPVD, echocardiography is sufficient for diagnosis and is usually preferable to cardiac catheterization. ${ }^{5}$ Identification of at least three veins by echocardiography is probably sufficient evidence to proceed with operation. As noted earlier, it is important to identify all four veins during operation. If, however, only two veins are identified by echocardiography, cardiac catheterization and angiocardiography should be considered to clarify the anatomy of pulmonary venous drainage. 
Magnetic resonance imaging is another imaging modality that may provide an accurate description of pulmonary venous drainage; early reports are promising but the clinical experience to date has been limited. ${ }^{28,29}$

Operative survivors after repair of mixed TAPVD appear to have long-term outcomes similar to those for other forms of TAPVD. An occasional patient may have late pulmonary vein stenosis, as seen in the other types of TAPVD. This stenosis may be at an anastomotic site or may be remote from the anastomosis, as was the case in the patient in our series with late pulmonary vein stenosis. The etiology of this form of pulmonary vein stenosis is unclear. Patients who survive correction of mixed TAPVD have excellent long-term functional results, with patients free of medications and enjoying normal activity.

In summary, mixed TAPVD is a comparatively rare variant of TAPVD. The primary challenges posed by these patients center around diagnosis and the technical aspects of surgical repair. The diagnosis and accurate description of anatomy are difficult to establish. Cardiac catheterization may be warranted if only two pulmonary veins are identified by echocardiography. Surgical approaches vary according to whether a $3+1$ or a $2+2$ drainage pattern is present. Operative survivors have good long-term survival and functional capacity, similar to those for other forms of TAPVD.

\section{REFERENCES}

1. Bando K, Turrentine MW, Sun K, et al. Surgical repair of total anomalous pulmonary venous connection: thirty-year trends [abstract]. Circulation 1995;92(Suppl 1):I51.

2. Cobanoglu A, Menasche VD. Total anomalous pulmonary venous connection in neonates and young infants: repair in the current era. Ann Thorac Surg 1993;55:43-9.

3. Serraf A, Bruniaux J, Lacour-Gayet F, et al. Obstructed total pulmonary venous return. J Thorac Cardiovase Surg 1991; 101:601-6.

4. Wilson WR Jr, Ilbawi MN, DeLeion SY, et al. Technical modifications for improved results in total anomalous pulmonary venous drainage. J Thorac Cardiovasc Surg 1992;103: $861-71$.

5. Lincoln CR, Rigby ML, Mercanti C, et al. Surgical risk factors in total anomalous pulmonary venous connection. Am J Cardiol 1988;61:608-11.

6. Turley K, Tucker WY, Ullyot DJ, Ebert PA. Total anomalous pulmonary venous connection in infancy: influence of age and type of lesion. Am J Cardiol 1980;45:92-7.

7. Lupinetti FM, Kulik TJ, Beekman RH 3rd, Crowley DC, Bove EL. Correction of total anomalous pulmonary venous connection in infancy. J Thorac Cardiovasc Surg 1993;106: $880-5$.
8. Sano S, Brawn WJ, Mee RB. Total anomalous pulmonary venous drainage. J Thorac Cardiovasc Surg 1989;97:886-92.

9. Raisher BD, Grant JW, Martin TC, Strauss AW, Spray TL. Complete repair of total anomalous pulmonary connection in infancy. J Thorac Cardiovasc Surg 1992;104:443-8.

10. de Leval MR, Stark J, Waterston DJ. Mixed type of total anomalous pulmonary venous drainage. Ann Thorac Surg $1973 ; 16: 464-70$.

11. Klindt R, Weldon C, Hartmann A Jr, et al. Mixed type total anomalous pulmonary venous drainage: report of a successful correction and review of the literature. J Thorac Cardiovasc Surg 1972;63:164-70.

12. Stark J. Anomalous pulmonary venous return and cor triatriatum. In: Stark J, de Leval MR, editors. Surgery for congenital heart defects. 2nd ed. London: WB Saunders, 1994:329-38.

13. Riegelman RK, Hirsch RP. Studying a study and testing a test: how to read the medical literature. Boston: Little, Brown, 1989:151-7.

14. Kraybill KA, Lucas RV Jr. Abnormal pulmonary venous connections. In: Emmanouilides GC, Riemenschneider TA, Allen HD, Gutgesell HP, editors. Heart disease in infants, children, and adolescents, including the fetus and young adult. 5th ed. Baltimore: Williams and Wilkins, 1995:838-47.

15. Neill CA. Development of the pulmonary veins, with reference to the embryology of anomalies of pulmonary venous return. Pediatrics 1956;18:880-7.

16. Darling RC, Rothney WB, Craig JM. Total pulmonary venous drainage into the right side of the heart: report of 17 autopsied cases not associated with other major cardiovascular anomalies. Lab Invest 1957;6:44-64.

17. Burroughs JT, Edwards JE. Total anomalous pulmonary venous connection. Am Heart J 1960;59:913-31.

18. Deslisle G, Ando M, Calder AL, et al. Total anomalous pulmonary venous connection: report of 93 autopsied cases with emphasis on diagnostic and surgical considerations. Am Heart J 1976;91:99-122.

19. Tajik AJ, Gau GT, Ritter DG, Schattenberg TT. Echocardiographic pattern of right ventricular volume overload in children. Circulation 1972;46:36-43.

20. Babb JD, McGlynn TJ, Pierce WS, Kirkman PM. Isolated partial anomalous venous connection: a congenital defect with late and serious complications. Ann Thorac Surg 1981; 31:540-3.

21. Saalouke MG, Shapiro SR, Perry LW, Scott LP 3rd. Isolated partial anomalous pulmonary venous drainage associated with pulmonary vascular obstructive disease. Am J Cardiol 1977;39:439-44.

22. Ports TA, Turley K, Brundage BH, Ebert PA. Operative correction of total left anomalous pulmonary venous return. Ann Thorac Surg 1979;27:246-9.

23. Van Meter Jr C, LeBlanc JG, Culpepper WS 3rd, Ochsner JL. Partial anomalous pulmonary venous return. Circulation 1990; (Suppl 4):IV195-8.

24. Brantigan $O$. Anomalies of the pulmonary veins. Surg Gynecol Obstet 1947;84:653-8.

25. Gustafson RA, Warden HE, Murray GF, Hill RC, Rozar GE, Partial anomalous pulmonary venous connection to the right side of the heart. J Thorac Cardiovasc Surg 1989;98:861-8.

26. Kirklin JW. Surgical treatment of anomalous pulmonary venous connections. Mayo Clin Proc 1953;28:476-9.

27. Bauer A, Korfer R, Bircks W. Left-to-right shunt at atrial 
level due to anomalous venous connection of left lung: report of seven cases. J Thorac Cardiovasc Surg 1982;84:626-30.

28. Choe YH, Lee HJ, Kim HS, Ko JK, Kim E, Han JJ. MRI of total anomalous pulmonary venous connections. J Comput Assist Tomogr 1994;18:243-9.

29. Wang JK, Li YW, Chiu IS, et al. Usefulness of magnetic resonance imaging in the assessment of venoatrial connections, atrial morphology, bronchial situs, and other anomalies in right atrial isomerism. Am J Cardiol 1994;74:701-4.

\section{Discussion}

Dr. Ralph S. Mosca (Ann Arbor, Mich.). Thank you for an interesting and well-presented article, and for the opportunity to review your manuscript before this session. The Great Ormond Street group has maintained excellent results for more than 20 years with this difficult group of patients with TAPVD. That only 20 patients were encountered at such a busy institution underscores the rarity of this lesion and the need for a high index of suspicion.

You have stressed the importance of an accurate preoperative diagnosis in planning a successful operation. This is especially true in neonates, in whom identifying these variants during operation may be difficult. Echocardiography, although highly specific, was only $31 \%$ sensitive in arriving at the correct diagnosis. Most would agree that leaving a single vein unrepaired is a wise decision if performing this repair risks pulmonary infarction. The resulting left-to-right shunt will be well tolerated in the great majority of cases. As a guideline in your manuscript, you stated that a cardiac catheterization may be unnecessary if three veins are well visualized and there is no clinical evidence of obstruction. I wonder whether other factors should also be considered. First, in situations in which there is dual drainage of a vein, one pathway may be serving to decompress this vein, and the presence or absence of obstruction may therefore be difficult to quantify.

In addition, the specific nonvisualized vein should be of some import as well. For example, given the fact that the right lower lobe vein is rarely anomalous in isolation, one can be reasonably certain of the diagnosis if all veins but the right lower lobe vein are seen. If the left lower lobe vein is not visualized, however, the exact configuration of the veins is much more uncertain. Perhaps you could comment on your indications for catheterization in light of these remarks.

Although it is necessary to perform an anastomosis of the left-sided veins to the left atrial appendage, as you have very nicely shown, the appendage is muscular, con- tracts with the cardiac cycle, and may be under some tension; it thus may have a tendency to stenose with time. You have excellent long-term follow-up, suggesting no major problems. Do you have any data concerning the patency of this anastomosis in long-term follow-up?

Finally, pulmonary hypertension remains a potentially serious problem in the postoperative management of these patients. What is your experience with the use of nitric oxide in this patient population?

It is encouraging to see the excellent long-term results that can be achieved if one can solve these initial structural problems. The results are a credit to your institutional expertise and innovation.

Dr. Delius. We actually wrestled quite a bit with the indications for cardiac catheterization. Christopher Lincoln, across town, wrote and article in the American Journal of Cardiology in 1988 that suggested that cardiac catheterization carries some significant morbidity in these patients. With the onset of color Doppler ultrasonography, I think that the echocardiographers are getting better in identifying all four veins and exactly where they connect. I think that in the end the decision to do cardiac catheterization has to be made individually for each patient. Some patients are going to be moribund before operation, and it would be difficult to perform a catheterization in those patients. But I think the issues that you point out are very poignant; if you have any questions, a cardiac catheterization would be quite reasonable.

You also raise an issue about which all of us have thought. Kirklin first did this left atrial appendage anastomosis in 1954, and I think people that have been asking about whether this causes stenosis or not ever since. There are no studies of which I am aware of that have documented whether this anastomosis stays patent. There is no clinically apparent stenosis evident, however, we do not know for sure. We were contemplating doing that as a next study.

In the article, we noted that three of our patients had postoperative pulmonary hypertension. These have been managed between 1971 and 1994, and there have been a large number of approaches used for the management of pulmonary hypertension. Nitric oxide was not used for any of these patients, primarily because most of those who had serious problems were operated on in the 1970s and 1980s. However, we have certainly used nitric oxide with great success for other forms of TAPVD. I believe that nitric oxide is helpful in the management of postoperative pulmonary hypertension. 